\title{
Maleficence and Non-beneficence of a Sedimentation Rate: A Lymphoma Case Analyzed from a Quaternary Prevention Perspective
}

\author{
Oliveira RM*, Silva C and Pires P \\ Lagoa Family Health Unit, Matosinhos Local Health Unit, \\ Portugal \\ *Corresponding author: Oliveira RM, Lagoa Family \\ Health Unit, Matosinhos Local Health Unit, Rua da Lagoa \\ s/n, 4450-352 Senhora da Hora, Matosinhos, Portugal
}

Received: March 21, 2018; Accepted: April 19, 2018; Published: April 26, 2018

\begin{abstract}
Screening tests should obey to well-defined criteria that assure that their application in asymptomatic patients results in more benefits than harms. The Erythrocyte Sedimentation Rate (ESR) is a blood test associated with severe diseases, but has low sensibility and specificity, and so it should not be used for screening purposes. In this case, the improper request of an ESR in a 48-years old man, previously healthy and asymptomatic, triggers an exhaustive cascade of tests that finally culminates in an oncologic diagnosis, but only established after the disease manifests clinically, that is, probably at the same stage as if no ESR has been dosed. On the contrary, the ESR request only resulted in the request of more tests, with more costs and potential associated risks, and in the development of an anxiety and sleep disorders that subsist even after the neoplasia remission. This case is illustrative of the iatrogenesis caused by the improper use of a test for screening purposes without any benefit in the early diagnosis and the importance of incorporating quaternary prevention in every medical intervention.
\end{abstract}

Keywords: Sedimentation rate; Lymphoma; Screening; Quaternary prevention

\section{Introduction}

Quaternary prevention was recently redefined as an action aimed to protect the patients from medical interventions that are likely of causing more harm than good [1]. This concept is particularly relevant when choosing a test for screening purposes. These should obey to well-defined criteria that assure their application results in more favorable outcomes (e.g. reduction in mortality) than adverse ones (over diagnosis and overtreatment) [2].

The Erythrocyte Sedimentation Rate (ESR) is a blood test that rises in infectious, inflammatory and neoplastic diseases, some of which are potentially severe. However, it also increases in some physiological states as pregnancy and aging, as well as in obesity or induced by drugs [3]. Since this, the ESR has a low sensibility and specificity, and its use in asymptomatic patients for screening purposes is not indicated $[3,4]$. Even so, according to a study conducted in Primary Care, $14 \%$ of all ESR requested were for screening purposes and $4 \%$ were requested by patient's demands [5].

This case report aims to demonstrate the tests cascade triggered by an improper ESR request in an asymptomatic patient and the maleficence that emerge from it, without any gain for an early diagnosis.

\section{Case Presentation}

48-years old man, electrical engineer and belonging to a highmedium social class. He smokes, has grade I obesity and arterial hypertension medicated with amlodipine $5 \mathrm{mg}$ and olmesartan $20 \mathrm{mg} /$ day. In routine blood tests, ordered by Occupational Medicine, it is identified an ESR of $30 \mathrm{~mm} / \mathrm{h}$, without any other laboratorial finding or symptom associated. In revaluation blood tests, 1 month after, the ESR is similarly increased, but now also with an increment of C-reactive protein, gamma-glutamyl transferase and alkaline phosphatase. These findings trigger an exhaustive etiological study with the request of serial blood tests, in which the same results remain. After 3 months, occurs an anemia of $11.2 \mathrm{~g} / \mathrm{dL}$, normochromic and normocytic, which keeps stable until the ending of the investigation. The vitamins, serum protein electrophoresis, autoimmune antibodies and serological tests were negative. It is also requested a chest $\mathrm{x}$-ray; prostate, renal, bladder and abdominal ultrasound exams; endoscopy and colonoscopy; pulmonary tuberculosis skin and blood tests, and transthoracic echocardiogram - all irrelevant.

After 4 months, the patient develops asthenia, night sweats and recurrent episodes of febricula - B symptoms - that the patient devalues by associating them with an anxiety disorder developed meanwhile. He starts having anxiety symptoms and insomnia after the performance of so many tests and the diagnostic uncertainty in which he was passing through, and so he was symptomatically medicated.

Then he is submitted to an abdominal computed tomography that reveals a hepatomegaly and enlarged retroperitoneal lymph nodes, indicating the realization of a positron emission tomography. This one suggests a lymphoproliferative disease and, in this stage, the physician starts to consider the hospital admission to perform a hepatic biopsy. That is when a palpable left supraclavicular lymph node of $2 \mathrm{~cm}$ comes up, which is biopsied and reveals the histopathological diagnosis of Hodgkin lymphoma, 8 months after the beginning of the study.
J Fam Med - Volume 5 Issue 2 - 2018

ISSN : 2380-0658 | www.austinpublishing group.com Oliveira et al. (C) All rights are reserved 


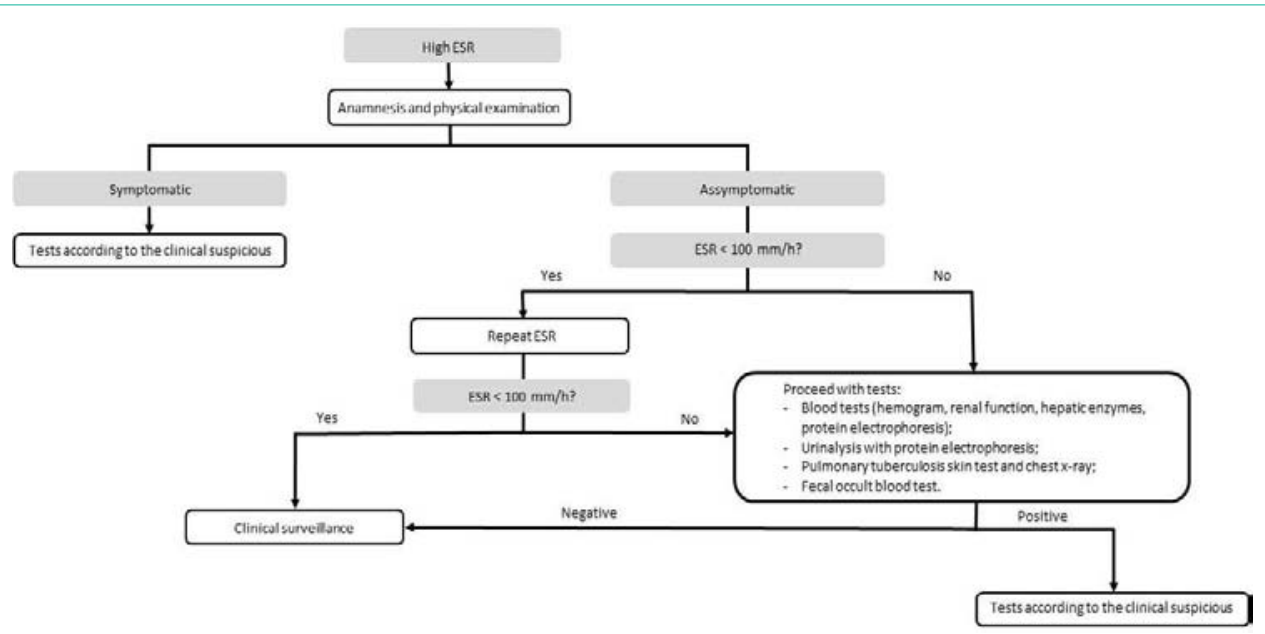

Figure 1: Flowchart for the management of an increased ESR based on ${ }^{3} \mathrm{e}^{4}$.

The patient is sent to the Oncology Department, where he completes 6 months of chemotherapy for a classic Hodgkin lymphoma in III-B stage. The psychiatric symptoms developed during the whole process become less evident with the definitive diagnosis, despite it being bad news. However, they did not revert in totally and 1 year after the disease remission he is still suffering for anxiety symptoms and insomnia with functional repercussion that demands antidepressant treatment.

\section{Discussion/Conclusion}

In this case, despite the early detection of a laboratorial finding and all the tests triggered to detect a potentially severe disease in an initial stage, the diagnosis was only established when the disease manifests clinically. That is, probably at the same stage as if no ESR has ever been dosed. Moreover, the patient interpretation of B symptoms as manifestation of his psychiatric disorder (reactive to the whole process), and its underestimation as an organic disease, could have even contributed to a diagnosis delay during the symptomatic phase.

The consequent tests cascade just brought about more costs (to the patient and to the healthcare system) and more potential associated risks. Moreover, the diagnostic uncertainty towards a severe diagnosis that last 8 months originated psychiatric symptoms, inducing a new health problem that was not present before. These symptoms, initially interpreted as an adaptation disorder reactive to a stress event, became a more sustainable problem in time and have required pharmacological intervention. Thus, it could be considered that the harmless request of an ESR has triggered a psychiatric disorder, probably in an already susceptible patient, but without functional impact until this event.

Our scientific literature research has not found case reports illustrating the iatrogenesis triggered by the improper use of a test, of which this case is well exemplificative.

We also have questioned what would be the right approach towards an isolated ESR increment. According to some authors, an
ESR less than $100 \mathrm{~mm} / \mathrm{h}$ should motivate the performing of a clinical history and physical exam and, if in the absence of clinical suspicious, the blood test should be repeated [3,4]. The interval duration is not established. If the subsequent ESR remains in the same values, there is no evidence to proceed to other investigations [3].

In the other hand, a markedly increased ESR of more than $100 \mathrm{~mm} / \mathrm{h}$ has a $90 \%$ of predictive value for severe disease [4]. A study conducted in ambulatory has concluded that $30.8 \%$ of these were caused by a malignancy, $21.7 \%$ by an infectious disease and $20.2 \%$ by an autoimmune disease [6]. In that case, it is recommended a clinical evaluation and tests according to the clinical suspicion, but if no obvious cause is identified, a limited number of tests are enough to reveal the cause as shown in Figure 1.

In conclusion, this case illustrates how an improper test request could not bring any beneficence for secondary prevention, but even more harms. It also emphasizes the importance of recognizing the characteristics of the different tests and always balancing risks and benefits in every medical intervention as accordance with a quaternary prevention approach.

\section{References}

1. Martins C, Godycki-Cwirko M, Heleno B and Brodersen J. Quaternary prevention: reviewing the concept. European Journal of General Practice. 2018; 24: 106-111.

2. Wilson $\mathrm{J}$ and Jungner $\mathrm{G}$. Principles and practice of screening for disease. Geneva: World Health Organization. 1968.

3. Brigden M. Clinical utility of the erythrocyte sedimentation rate. Am Fam Physician. 1999; 60: 1443-1450.

4. SOX H. Diagnostic Decision: The Erythrocyte Sedimentation Rate: Guidelines for Rational Use. Annals of Internal Medicine. 1986; 104: 515.

5. Grønlie M and Hjortdahl P. The Erythrocyte Sedimentation Rate; Its Use and Usefulness in Primary Health Care. Scand J Prim Health Care. 1991; 9: $97-$ 102.

6. Otero-Castro V, Bonella B, Cristaldo N, Fiorentini, F, Giunta D, Massimino $\mathrm{B}$, et al. Extremely elevated erythrosedimentation in an adult population in the City of Buenos Aires. Revista chilena de infectología. 2017; 34: 314-318. 